Full Length Article

\title{
Flexible, multifunctional nanoribbon arrays of palladium nanoparticles for transparent conduction and hydrogen detection
}

\author{
Elena H. Sánchez ${ }^{\mathrm{a}, \mathrm{b}}$, Peter S. Normile ${ }^{\mathrm{b}}$, Jose A. De Toro ${ }^{\mathrm{b}}$, Rubén Caballero ${ }^{\mathrm{a}}$, J. Canales-Vázquez ${ }^{\mathrm{c}}$, \\ Esther Rebollar ${ }^{\mathrm{d}}$, Marta Castillejo ${ }^{\mathrm{d}}$, Jose M. Colino, ${ }^{\mathrm{a}, *}$ \\ ${ }^{a}$ Instituto de Nanociencia, Nanotecnología y Materiales Moleculares and Depto. de Física Aplicada, Universidad de Castilla-La Mancha, Campus de la Fábrica de Armas, \\ 45071 Toledo, Spain \\ ${ }^{\mathrm{b}}$ Instituto Regional de Investigación Científica Aplicada and Depto. de Física Aplicada, Universidad de Castilla-La Mancha, 13071 Ciudad Real, Spain \\ ${ }^{\mathrm{c}}$ Instituto de Energías Renovables, Universidad de Castilla-La Mancha, 02006 Albacete, Spain \\ ${ }^{\mathrm{d}}$ Instituto de Química-Física Rocasolano, Consejo Superior de Investigaciones Científicas Serrano 119, 28006 Madrid, Spain
}

A R T I C L E I N F O

\section{Keywords:}

Laser induced periodic surface structures

Oblique deposition

Nanoparticles

Palladium

Transparent conductors

Hydrogen detection

\begin{abstract}
A B S T R A C T
A novel combination of nanostructuring techniques is undertaken to obtain a multifunctional material system consisting of planar arrays of nanoribbons of Pd nanoparticles (NPs) on polyethylene terephthalate (PET) substrates. The first stage was to prepare a laser-induced periodic surface structure (LIPSS) on the PET substrate, which electron microscopy revealed to be a large area, high coherence nanoripple pattern with spacing of $(204 \pm 1) \mathrm{nm}$ and amplitude of $(46 \pm 9) \mathrm{nm}$. The second stage comprised NP (diameter $5 \mathrm{~nm}$ ) deposition by orientating the nanostructured substrate to a Pd NP beam from a sputter gas phase aggregation source such that the substrate was partially shadowed by the ripple pattern (the incident angle with respect to the normal of the PET substrate was varied between 60 and $75^{\circ}$ ). This resulted in the formation of an array of NP ribbons (thickness $\sim 20 \mathrm{~nm}$ ) on the ripple ridges, the mean ribbon width depending on the deposition incidence angle, thus confirming the shadowing effect. These planar arrays were studied as candidates for both flexible, transparent conductors and hydrogen sensors. Analysis of optical transmittance indicates that a mean inter-ribbon gap of above $100 \mathrm{~nm}$ is required in order to improve the average transmittance beyond $80 \%$. Four-probe electrical resistance measurements show these nanoribbon arrays to be electrically anisotropic structures whose sheet resistance is understood to be governed by the contact resistance between NPs. An additional functionality is proven for the fabricated substrates: reversible detection of hydrogen at a partial pressure above $\sim 60 \mathrm{ppm}$, with good electrical sensitivity in the dilute ( $\alpha$-hydride) regime.
\end{abstract}

\section{Introduction}

Several future technological applications based on nanostructures entail novel fabrication methods together with specific performance requirements $[1,2]$. For example, nanostructured materials for some advanced electronics and optoelectronics applications are synthesized on lightweight, flexible and transparent substrates such as light polymer films. The use of polymer substrates, however, also brings challenges. For the next generation of transparent conductors (TCs), several groups have investigated hybrid materials prepared by low temperature solution processing and large area deposition techniques. Random meshes of metallic nanowire networks (MNN) have become the most promising alternative to traditional TC because their properties include flexibility and stretching capability [3]. Another materials property to address is electrical transport anisotropy. Several applications require the use of anisotropic transparent conductors, such as the active components in liquid crystal displays used for the alignment of calamitic mesogens [4]. Alignment of liquid crystals produces a high birefringence behavior when they are exposed between crossed polarizers. Other common applications are electrodes for sensors and solar cells, all of which are often based on indium tin oxide (ITO), the most popular TC in industry because of its low resistivity $\left(\sim 10^{-4} \Omega \mathrm{cm}\right)$, high transmittance (80-90\%) and low deposition temperature. Despite the performance of ITO films deposited on polymer substrates being inferior to that of ITO films deposited on glass substrates, ITO thin films on polyethylene terephthalate (PET) have an excellent combination of optical transmittance ( $80 \%$ in the visible range) and low sheet resistance $(\mathrm{Rs}=173 \Omega / \mathrm{sq}$ ) [5]. However, both the need to replace the

\footnotetext{
* Corresponding author.

E-mail address: josemiguel.colino@uclm.es (J.M. Colino).
} 
indium, systematically listed by the European Commission as a critical raw material [6], and ITO's brittleness (and propensity to crack when subject to bending) have prompted the search for other alternative TCs.

In addition to obtaining new, flexible and anisotropic TCs, it is interesting to attempt to achieve multifunctionality in a single material system. Visible light transmittance, electrical transport and gas (in particular, hydrogen) sensing properties is an appealing combination of functionalities. Widespread use of hydrogen as a fuel will require innovations in hydrogen storage and sensing. The growing hydrogen infrastructure calls for the development of compact, reliable and safe $\mathrm{H}_{2}$ sensors for the environments of both the supplier and the end-user. Palladium is widely used as a sensing material for $\mathrm{H}_{2}$ due to its ability to absorb a large amount of this gas at ambient conditions, particularly in the form of thin films [7,8]. When Pd is exposed to $\mathrm{H}_{2}$ gas, molecules of the latter are adsorbed onto the metal surface and dissociate into hydrogen atoms. Bulk diffusion of these hydrogen atoms leads to their occupation of interstitial sites of the Pd lattice, causing a lattice expansion and an electrical resistivity increase as a Pd hydride is formed. In the case of bulk samples, formation of the $\alpha$-hydride phase occurs at up to $\sim 200 \mathrm{ppm} \mathrm{H}_{2}$ and the (cubic) lattice structure expands slightly $(<0.13 \%)$. However at concentrations around $\sim 1000 \mathrm{ppm}_{2}$, the $\alpha$ hydride phase starts to convert into the $\beta$-hydride phase, which is associated with a larger lattice expansion $(>3 \%)$ and resistivity increase. According to Sakamoto et al. [9], the variation in resistance with hydrogen content may be mainly associated with an incomplete formation of the $\beta$-hydride, i.e., to a coexistence of the $\alpha$ and $\beta$ phase (two-phase region). Remarkably, it is found that various Pd nanostructures show better performance than bulk Pd in terms of fast response, high sensitivity, high $\mathrm{H}_{2}$ selectivity, room temperature operability, lower detection limit and low power consumption. Nanostructures allow an increased surface area-to-volume ratio which may increase the sensing performance. Furthermore, the use of nanoparticles (NPs) as building blocks further enhances this ratio due to the formation of a porous, electrically semi-continuous morphology. Joshi et al. [10] demonstrated that Pd NP films have better hydrogen sensitivity than continuous films of similar thickness. Nonetheless nano-sized materials, particularly NPs, show significant changes in equilibrium properties as compared to their bulk counterparts. The NP hydrogen-palladium system shows deviations from the bulk pressure-H isotherms, shifted phase boundaries, narrowing of the $\alpha+\beta$ two-phase region [11] and possibly a different lattice structure for Pd clusters (diameter $\sim 5 \mathrm{~nm}$ ) with large hydrogen loading [12]. Beyond the effect of an increased surface-to-volume ratio in semi-continuous nanoparticle films, the accessibility of Pd nanostructures to $\mathrm{H}_{2}$ is best achievable by surface patterning. In addition, patterning of nanowire or nanoribbon arrays may allow anisotropic electrical conduction and simultaneously contribute to relieving large internal mechanical stress in the Pd-H system.

In the present work we investigate a flexible nanostructured material fabricated by means of the novel sequential combination of laserbased surface patterning of a polymer substrate and oblique deposition of a nanoparticle beam onto the patterned surface. The first stage of sample fabrication is based on high spatial resolution patterning of soft polymeric matter using the high throughput technique of laser induced periodic surface structures (LIPSS). This method has previously been successfully applied using a polarized laser source at several laser wavelengths and pulse durations [13]. The method does not require cleanroom facilities. In the second fabrication stage the modulated LIPSS surface acts as a template for self-shadowing of an obliquely incident NP beam. The use of palladium as the NP material is shown to lead to anisotropic conductors comprising planar arrays of aligned nanoribbons of these particles with potentially dual applications as transparent conductors and hydrogen sensors.

\section{Materials and methods}

The polymeric substrates ( $125 \mu \mathrm{m}$ thick, free-standing film) were nanostructured by irradiation at normal incidence with a linearly polarized laser beam from a Q-switched Nd:YAG laser (Lotis TII LS$2131 \mathrm{M}$ ) with pulse duration of $8 \mathrm{~ns}$ at a repetition rate of $10 \mathrm{~Hz}$. The fourth harmonic of the fundamental laser radiation at $266 \mathrm{~nm}$ was used due to the high light absorption coefficient of PET at this wavelength. Formation of LIPSS was achieved at optimum conditions of fluence and number of pulses. Laser fluence was determined by measuring the laser energy in front of the sample and calculating the area of the irradiated spots after delimitating the beam with an iris of $0.45 \mathrm{~cm}$ diameter. Laser fluence typically ranges $8-10 \mathrm{~mJ} / \mathrm{cm}^{2}$. Samples were mounted on a motorized XY translation stage and in order to irradiate larger areas a sample scanning process was used. The scanning speed, and consequently the spatial overlap of successive pulses, was chosen to apply the optimal amount of pulses for LIPSS formation, which exceeds 3600 . The fluence must reach a minimum value to overcome the glass transition temperature on the surface of the amorphous polymer, but are well below the ablation threshold of PET at this wavelength of irradiation [13].

The palladium NPs were synthesized using a sputter gas phase aggregation source operated at a dc power of $50 \mathrm{~W}$ and employing a 6:1 ratio of Ar:He atmosphere at a pressure of approximately 0.2 mbar. The NPs were injected through a pin hole into the main (deposition) chamber, where they landed on the patterned polymeric substrate (Fig. 1(a)). The deposition process was carried out at a vacuum of $4 \times 10^{-3}$ mbar. The deposition rate and thickness were measured by using a quartz crystal thickness monitor. During deposition, the substrates were placed over a triangular prism face, which enabled control of the incidence angle $(\theta)$ between the nanoparticles beam and the normal to the substrate (Fig. 1(b)). The value of this angle was critical for obtaining well-defined nanoribbons as well as controlling the ribbon width. The nanoribbons array structure was formed due to the selfshadowing effect of the ripple surface. A set of array samples was grown at different deposition angles with fixed ribbon thickness of $20 \mathrm{~nm}$. To serve as a control, another set of Pd films were grown but this time at normal incidence on flat PET substrates, varying the film thickness for use in optical transmittance measurements.

The nanostructured surfaces were characterized by using atomic

(a)
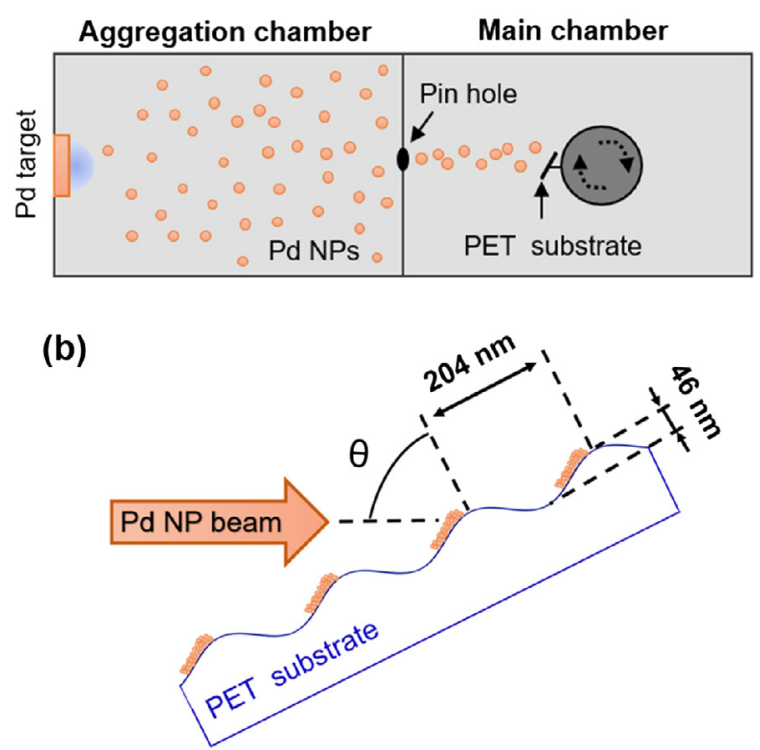

Fig. 1. (a) Layout of the NP deposition apparatus, consisting of a sputter gas phase aggregation source (left side) for NP fabrication and the main chamber (right side) equipped with a rotary substrate holder for oblique deposition of the Pd NP beam that emerges from the pin hole. (b) Cross-section of nanoscale LIPSS and nanoribbons formed by oblique deposition. The angle of incidence $(\theta)$ is defined with respect to the normal to the average substrate surface. 
force microscopy (AFM, Nanosurf) in dynamic and phase contrast mode before and after the NPs deposition. The images were analysed using the software Gwyddion 2.49. The period and amplitude of the ripples were determined from AFM topographic analysis. The average period was calculated for the whole topography image by using the Fast Fourier Transform (FFT) profile but other pattern dimensions were also computed from histograms and line profiles. Scanning electron microscopy (SEM) analysis was carried out using a GeminiSEM 500 (Zeiss), which is equipped with a field-emission gun and was operated at an accelerating voltage between 1 and $2 \mathrm{kV}$. SEM images were taken to investigate the NP ribbons morphology. The same equipment was also used for energy dispersive X-ray microanalysis (EDX), employing an accelerating voltage of between 10 and $20 \mathrm{kV}$ to obtain compositional images of the samples. Transmission electron microscopy (TEM) was performed using a Jeol JEM-2100, studying Pd NPs deposited onto $\mathrm{Cu}$ grids using the same sputter source operation conditions as above. Xray diffraction was carried out using a Bruker D8 diffractometer, employing a transmission geometry to study thick $(>100 \mathrm{~nm})$ films of Pd NPs deposited onto mica substrates (using, again, the same sputtering conditions). UV-visible transmittance spectra were recorded with a Shimadzu UV 1603 spectrophotometer using unpolarised light in the wavelength range $300-900 \mathrm{~nm}$. Spectral transmittance of Pd samples were obtained after correcting for the transmittance of the PET substrate. The electrical resistance was measured using a four-probe method. The measurements were carried out with a programmable current source and nanovoltmeter combination (Keithley $6221+2182 \mathrm{~A}$ ). The samples were cut into rectangular shapes and four silver pads were painted in a row on the large side to provide electrical contact for thin copper wires. A custom-built experimental setup was used for hydrogen sensing experiments. The samples with the silver contacts were placed into a small chamber fitted with a gas inlet, a vacuum connection and electrical feedthroughs. The static gas pressure was controlled manually with a screw valve. The electrical resistance was measured while each sample was exposed from a vacuum environment up to a fixed pressure of a $1 \% \mathrm{H}_{2} / \mathrm{N}_{2}$ gas mixture, and then back to vacuum. Fixed pressure values in the range from $10^{2}$ to $10^{5} \mathrm{~Pa}$ have been employed. All data acquisition was performed with a LabView software program.

\section{Results and discussion}

\subsection{Microscopic characterization}

AFM and SEM both indicate a highly coherent ripple pattern on the patterned polymer surface, before and after Pd deposition. The micrographs in Fig. 2(a) and (b) are exemplars of those obtained with these respective techniques after oblique NP deposition. A couple of Y-shaped defects are highlighted by the red circles in Fig. 2(a). Given such defects, the ribbons' longitudinal coherence is estimated to be $10 \mu \mathrm{m}$. The 'Y-shape' defects can be observed randomly distributed on the substrate before the Pd nanoparticles deposition. They appear during the LIPSS formation and they are presumably due to several factors, such as initial inhomogeneities in the polymeric substrate or misorientation of laserbeam scanning direction in relation to the to light polarization direction $[14,15]$. Several approaches have been tested by other researchers to increase the regularity of LIPSS patterns, including simultaneous irradiation with two harmonic wavelength [16] but, in this work, we have not implemented any such strategies to try decrease the number of Yshaped defects. The Pd NPs possess the (bulk) Pd FCC crystal structure as determined by X-ray diffraction (no other crystallographic phase is detected) and a mean diameter of around $5 \mathrm{~nm}$ obtained by TEM [see the upper inset of Fig. 2(a)].

Ribbon width, ripple spacing and amplitude could be measured with histograms collected from a number of topography line profiles [the lower inset of Fig. 2(a) shows an example]. A mean amplitude of $(46 \pm 9) \mathrm{nm}$ is determined from AFM topography images such as that
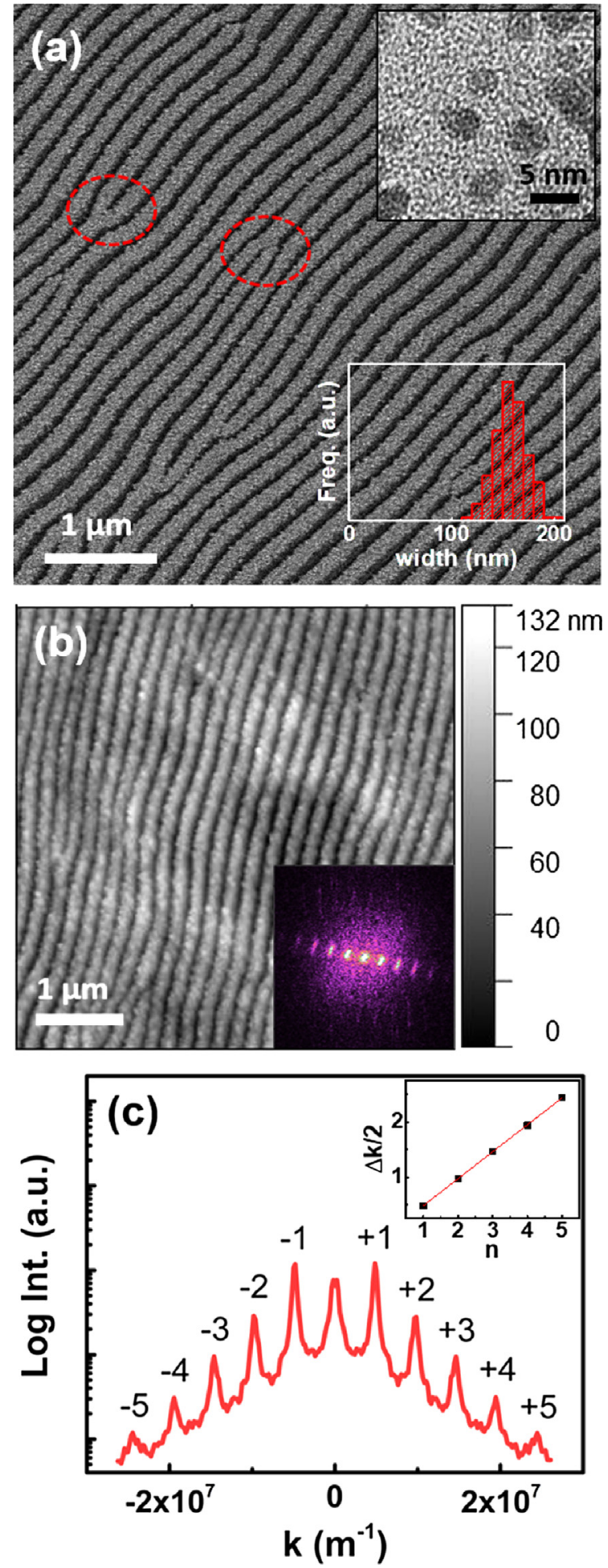

Fig. 2. (a) $6 \mu \mathrm{m} \times 6 \mu \mathrm{m}$ SEM image of a nanoribbon array deposited at $\theta=65^{\circ}$. Red circles mark Y-shaped defects. The upper inset is a TEM image of the Pd nanoparticles deposited on a copper (TEM) grid. The lower inset is a histogram of ribbon widths from which mean values can be obtained. (b) $5 \mu \mathrm{m} \times 5 \mu \mathrm{m}$ AFM topographic image of the same sample as in panel (a). The inset shows a 2D-FFT of this topography image showing interference orders in the transverse direction plus a central diffuse halo. (c) Line profile of 2D-FFT along the direction transverse to LIPSS. Positive and negative interference orders are numbered. The inset shows a linear fit to the reciprocal distance $(\Delta \mathrm{k} / 2)$ as a function of order number of interference maximum $(n)$, which has been used for the precise measurement of mean LIPSS spacing. $\Delta \mathrm{k} / 2$ has units of $10^{7} \mathrm{~m}^{-1}$. 
in Fig. 2(b). These micrographs also show a ripple pattern with high transversal coherence as indicated by the FFT image in the inset of Fig. 2(b), which presents up to five interference maxima in the direction across the pattern, and by the line profile of the same image, shown in Fig. 2(c). This profile has been used to precisely obtain the mean ripple spacing through a linear fit of the reciprocal length position of each maximum (with respect to the central maximum, $\Delta \mathrm{k} / 2$ ) as a function of the interference order [inset of Fig. 2(c)]. A mean spacing of (204 \pm 1$)$ $\mathrm{nm}$ is obtained from the inverse of the slope. Both ripple spacing and amplitude are critical parameters to produce the required shadow effect for the ribbon formation, determining a minimum incidence angle for the appearance of shadowed regions (for a sinusoidal profile) $\theta_{\min } \approx 55^{\circ}$, and thus the desired NP stripes $\left(\theta_{\min }\right.$ is estimated as being the complementary angle to the arctangent of the maximum slope of the sine wave in Fig. 1(b) with respect to a flat substrate surface). The isotropic halo observed in the FFT image in the inset of Fig. 2(b) - a feature not observed in the FFT image of the bare LIPSS (not shown) arises due to the surface morphology associated with nanoparticles randomly distributed along the ripples. The interference maxima are observed in the FFT image before and after NP deposition, indicating that the rippled pattern is not undermined by the deposition process.

Ribbons of Pd NPs deposited at different incidence angles are shown in Fig. 3. The mean ribbon width was estimated using histograms obtained from several SEM images of each sample. As expected for selfshadowing, a decrease in the width with higher incidence angles is evident from Fig. 3(d). Incidence angles below $\theta_{\min }$ will result in a continuous film of non-uniform thickness. On the other hand, for $\theta$ approaching $90^{\circ}$ (i.e. grazing incidence) the effective deposition rates $(\propto \cos \theta)$ will be impractically low.

Images obtained using EDX (not shown) confirm the presence of Pd only within the ribbons. Furthermore, analysis of AFM images recorded in dynamic and phase contrast modes confirms the shadow effect through the contrast between the Pd stripes and the intercalated shadowed PET.

\subsection{Optical transmittance and electrical conductivity}

In order to determine the optical characteristics of the Pd NP ribbon arrays, we first prepared a set of Pd NP films of different thickness deposited on flat (i.e., without LIPSS) PET substrates. Microscopy of these samples confirmed a morphology of Pd NPs randomly covering each surface. The effective Pd thickness is below the average particle diameter $(5 \mathrm{~nm})$ in two films, namely 1 and $2 \mathrm{~nm}$ respectively, hence the films are discontinuous. A third film of larger effective thickness $(10 \mathrm{~nm})$ was found to present a continuous surface morphology. Fig. 4(a) shows the spectral transmittance of the bare flat PET substrate together with that of the three Pd NP films. The Pd transmittance values have been corrected for the substrate in each case. No evidence of plasmon resonance is found in any of the curves. The wavelengthaveraged transmittance of the continuous $(10 \mathrm{~nm})$ NP film amounts to only $50 \%$, below the expected value $(\sim 80 \%)$ for such thickness if we compute the transmittance using a skin depth of $50 \mathrm{~nm}$ for pure Pd [17], which may point to a reduced effective skin depth in NP samples.

Fig. 4(b) shows the spectral transmittance of the bare rippled PET substrate and of the Pd NP ribbons (after correcting for the rippled PET substrate). Both the flat and rippled surface PET display a transmittance of around $90 \%$ in the visible range without significant structure. Only for wavelengths below $460 \mathrm{~nm}$ and close to the polymer absorption edge does the ripple structure present a lower transmittance compared to the flat PET substrates. The three nanoribbon arrays, prepared at different oblique incidence angles, all comprise approximately the same ribbon thickness of $\sim 20 \mathrm{~nm}$ but have different ribbon widths. The dependence of the transmittance of the nanoribbon arrays at the wavelength of $550 \mathrm{~nm}$ (this wavelength has been chosen for comparison with results by several other authors $[18,19]$ ) on the ribbon width is shown in the inset of Fig. 4(b). With this dependence we can estimate, by a
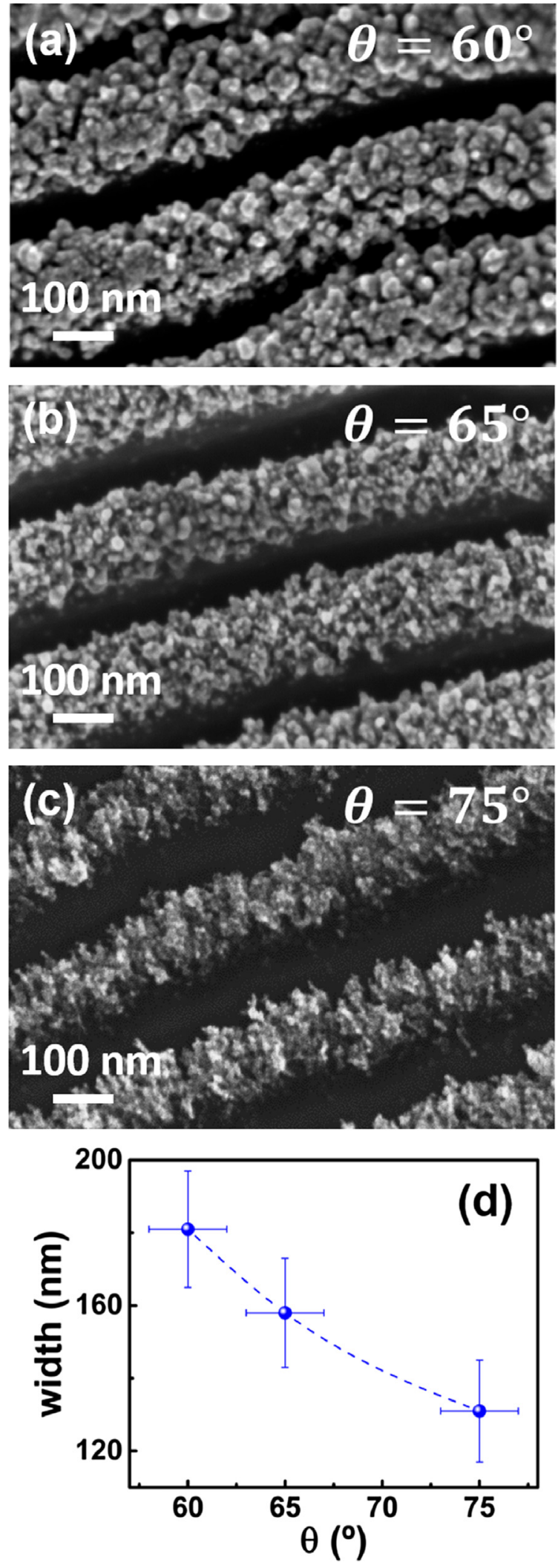

Fig. 3. (a), (b) and (c): SEM images of Pd nanoribbon arrays grown at incidence angles of $60^{\circ}, 65^{\circ}$ and $75^{\circ}$ respectively. A higher incidence angle makes oblique deposition of ribbons with reduced width. (d) Plot of mean nanoribbon width as a function of the incidence angle. The dashed line is a guide to the eye. 
(a)

(b)
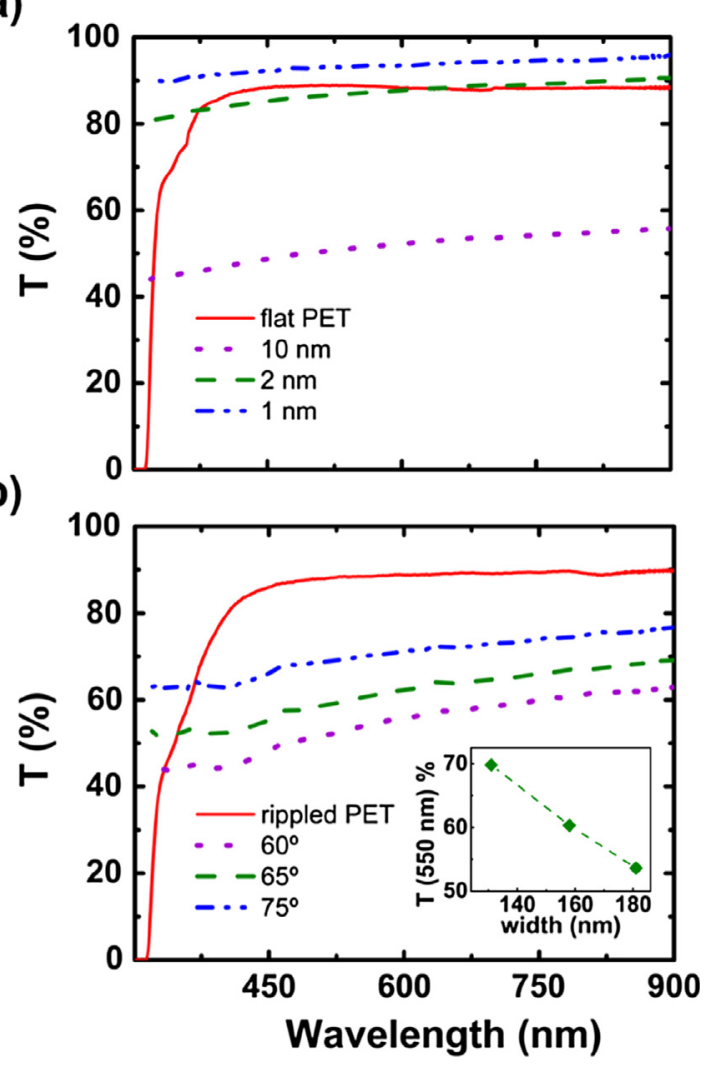

Fig. 4. (a) Optical transmittance of nanoparticle Pd films of different thickness on flat PET substrates. The solid line corresponds to data for the bare substrate. (b) Optical transmittance of nanoribbon arrays made on LIPSS for three samples corresponding to different incidence angles $\left(60^{\circ}, 65^{\circ}\right.$ and $\left.75^{\circ}\right)$. Again, the transmittance of the bare (this time rippled) PET substrate is shown for comparison (solid line). The inset plots transmittance at a fixed wavelength $(550 \mathrm{~nm})$ as a function of mean ribbon width for the three samples. The Pd transmittance in both panels correspond to experimental values after correction for the substrate in each case.

linear extrapolation, that the transmittance would reach values above $80 \%$ if the ribbon width was reduced to $100 \mathrm{~nm}$, which could be realized by using somewhat larger incidence angles. If a particular application of planar arrays of nanoribbons required transmittances above $\sim 85 \%$, a strategy could be to pattern the polymer surface with a longer laser wavelength in order to achieve a larger spacing compared to wavelengths in the visible range. The LIPSS technique actually offers the possibility of tuning the pattern spacing and also the use of a different polymer, which may be tailored to a particular application [13]. Catrysse et al. [20] investigated the theoretical transmittance of 1D nanopatterned metallic films as transparent electrodes, studying the normal incidence, average transmittance of a periodic arrangement of metallic wires (spacing $400 \mathrm{~nm}$ ) as a function of wire width, which was found, interestingly, to decrease rapidly for widths above $100 \mathrm{~nm}$. Although the nanoribbon arrays described here are not perfect 1D gratings, it is likely that the ribbon widths [see inset of Fig. 4(b)] are too large to expect high (80-90\%) transmittance values.

Other materials systems have been explored by several groups and reach a high transmittance. Especially remarkable is the very high transmittance of metallic nanowire networks (MNN) achieved mostly by passing the light through the (random) network gaps, as light with a wavelength shorter than the gap size can be readily transmitted [21]. Fig. 5 shows the comparison of optical transmittance (at $550 \mathrm{~nm}$ ) and sheet resistance of representative systems used as TCs, including continuous metallic film and MNNs, together with the Pd NP systems described here. The transmittance of the $10 \mathrm{~nm}$ thick continuous Pd NPs

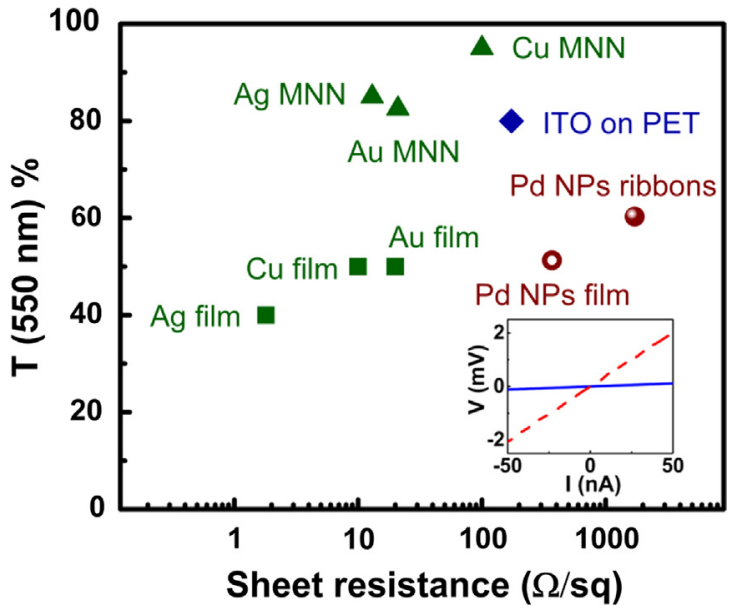

Fig. 5. Comparison of optical transmittance at a fixed wavelength $(550 \mathrm{~nm})$ as a function of electrical sheet resistance for various flexible, transparent conducting materials including the fabricated nanoribbon arrays. Metallic continuous films (squares) [18,19,23], metallic nanowire networks (triangles) $[18,22,24]$, ITO on PET (diamond) [5], Pd nanoparticles film (open circle) and Pd NPs ribbons (closed circle). The inset shows DC Voltage-Current curves of one Pd nanoribbon array $\left(\theta=65^{\circ}\right)$ measured with current either parallel (solid line) or perpendicular (dashed line) to the pattern direction.

film is similar to that of the other metallic thin films (Ag, $\mathrm{Cu}$ and $\mathrm{Au}$ films of thickness 25,50 and $7 \mathrm{~nm}$ respectively), i.e. values below around $50 \%$. For the continuous metal films, the transmittance is directly related to the skin depth, which is approximately a few tens of nanometers at optical frequencies, and the sheet resistance is optimally low. A better transmittance of these continuous systems would require smaller thickness, which, as a trade-off, would lead to enhanced sheet resistance. A very promising alternative for transparent conductor materials is the MNN approach [3]. The Cu MNN shown in Fig. 5, with $35 \mathrm{~nm}$ thickness, achieved the best transmittance $(\mathrm{T}=95 \%)$ and a sheet resistance $\mathrm{R}_{\mathrm{S}}=100 \Omega / \mathrm{sq}$ [22], but it remains a challenge to stabilize the $\mathrm{Cu}$ nanowires against oxidation. The best sheet resistance has been achieved by Ag MNN with $160 \mathrm{~nm}$ thickness [18].

The sheet resistance of the Pd NP nanoribbon arrays described here $(\sim 3 \mathrm{~m} \Omega \mathrm{cm})$ is higher than the other MNN systems due to several factors. Firstly, the electrical resistivity of pure palladium is $5-6$ times higher than that of silver or copper, common metals for lithographed interconnections or metallic networks previously discussed. Secondly, the nanoparticle morphology of nanoribbons will imply a significant porosity (volume filling factor of around 0.5 at the most). Thirdly, the nanoparticle morphology induces a large contact resistance between every pair of particles and, accordingly, to a large total resistance. These latter two factors will also account for the very high resistivity measured in Pd nanoribbon arrays as compared with that of pure Pd $(\sim 0.01 \mathrm{~m} \Omega \mathrm{cm})$. Catrysse et al. [20] demonstrated the strong influence of the geometrical parameters of 1D nanopatterned metallic films on the electrical properties. As expected, those authors found that an increase in the wire width, at fixed wire thickness, results in a decrease in the electrical sheet resistance. However, those authors (as well as the present study) also find an increased width to result in a reduction in the optical transmittance of the system. Thus, the realization of nanopatterned metallic films of a constant sheet resistance and high optical transmittance will involve designing narrow but thick wires.

The inset of Fig. 5 plots current-voltage data at room temperature for two different pieces of the same Pd nanoribbon array $\left(\theta=65^{\circ}\right)$. One curve was measured with the current applied parallel to the pattern direction while the other was obtained with the current applied perpendicular to the pattern direction. Both data sets are linear as expected for ohmic semi-continuous films, however the slope (resistance) depends strongly on the current direction. After the calculation of 
resistivity for each configuration, we obtain a transport anisotropy $\rho_{\perp} /$ $\rho_{\text {II }} \approx 25$. Such a high ratio is desirable for the control of directional electric fields in future devices, adding a notable functionality to the present system absent in the electrically isotropic MNNs commented above. The width (and thickness) of Pd nanoribbons certainly shape the electrical resistivity but would not have any effects to the transport anisotropy if the nanoribbon pattern was perfectly defined because the anisotropy is the ratio of both configurations $\rho_{\perp} / \rho_{\mathrm{II}}$. However, the ohmic conductivity in the (high-resistance) perpendicular configuration results from the presence of Y-shaped defects in the LIPSS pattern (see Fig. 2(a) and Section 3.1) leading to electrical connections between the NP stripes. Thus, we believe that transport anisotropy is governed by the density of defects in the LIPSS pattern, which replicates on the ribbon pattern causing a diminished anisotropy ratio. In fact, the perpendicular resistivity may be generally used as a measure of the density of such defects in LIPSS.

\subsection{Hydrogen detection}

In order to test the hydrogen-sensing capability of our nanoribbon arrays, we have measured the electrical sensitivity, defined as $S=\Delta R$ / $\mathrm{R}_{0}$, at a range of static pressures of a $1 \% \mathrm{H}_{2} / \mathrm{N}_{2}$ gas mixture, from $10^{2}$ to $10^{5} \mathrm{~Pa}$. Fig. 6 shows sensitivity data obtained from reversible cycles, where the lowest detectable limit corresponds to an equivalent $\mathrm{H}_{2}$ concentration in ambient conditions of approximately $60 \mathrm{ppm}$. The percentage sensitivity $\left(100 \mathrm{~S}\right.$ ) reaches a value of $4.6 \%$ at $\left[\mathrm{H}_{2}\right]=1 \%$ in atmospheric pressure (equivalent concentration of around $10^{4} \mathrm{ppm}$ ), which quantitatively compares well with other types of pure Pd based sensors at room temperature, such as nanowire networks in filtration membranes [25] $\left(\mathrm{S} \approx 5 \%\right.$ at $\left.\left[\mathrm{H}_{2}\right]=1 \%\right)$, nanoparticle layers [26] (S $<15 \%$ at $\left.\left[\mathrm{H}_{2}\right]=100 \%\right)$ and single nanowires [27] ( $\mathrm{S}=10-12 \%$ at $\left[\mathrm{H}_{2}\right]=1 \%$ ). Rumiche et al. [28] reported $\mathrm{S}$ ranging from 0.5 to $3 \%$ at $\left[\mathrm{H}_{2}\right]=1 \%$ for Pd nanowire arrays grown in membranes.

The inset of Fig. 6 shows the electrical sensitivity under two cycles of hydrogen exposure at fixed pressure, confirming reversible behavior and reproducibility, both essential requirements for sensor operation. The diffusion of hydrogen into thick continuous Pd films may result in a large internal stress, leading to film buckling for thickness $>20 \mathrm{~nm}$ [25]. Such irreversible deformation leads to an irreversible resistance change, hence eliminating reusability. SEM and AFM investigations of our nanoribbon arrays do not show any buckling or width change upon hydrogen cycling. This result can be understood from the relatively low $\mathrm{H}_{2}$ loadings used in our experiments, insufficient to drive the $\mathrm{Pd}-\mathrm{H}$

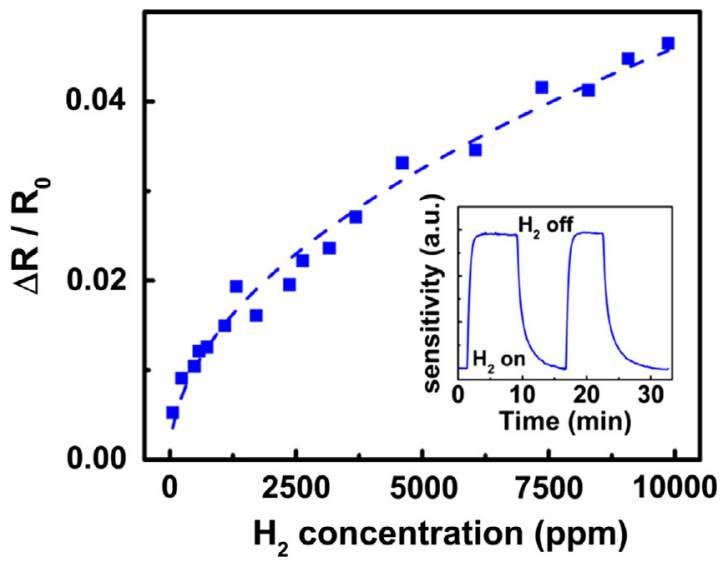

Fig. 6. Change of electrical resistance upon hydrogen cycling, $\Delta \mathrm{R}$, normalized to the initial resistance $\mathrm{R}_{0}$ before $\mathrm{H}_{2}$ exposure, as a function of equivalent $\mathrm{H}_{2}$ concentration in ambient conditions. The dashed line is a fit to Sievert's law. The inset shows the time evolution of the resistance in the Pd nanoribbon array $\left(\theta=65^{\circ}\right)$ for two hydrogen cycles up to the same static pressure $\left(10^{5} \mathrm{~Pa}\right)$ but different exposure times. nanoparticles into the $\beta$-hydride phase (which is known to exhibit very large lattice expansions). Indeed, small Pd nanoparticles present an enhanced solubility in comparison with the bulk [11], thus the maximum hydrogen content of 10,000 ppm employed here would not be enough to initiate the conversion of $\alpha$-hydride into $\beta$-hydride phase [12]. In well-annealed bulk palladium, the initial dilute phase hydrogen solubility obeys Sievert's law, i.e. plots of $\left(\mathrm{pH}_{2}\right)^{1 / 2}$ against the hydrogen content $[\mathrm{H}] /[\mathrm{Pd}]$ are linear with a characteristic slope intersecting the origin as $[\mathrm{H}] /[\mathrm{Pd}]$ tends to zero [29]. Deviations from this behaviour must be due to the presence of defects of all types, especially extended defects in bulk Pd, which are common diffusion paths. The absorbed hydrogen atoms interact with $\mathrm{Pd}$ atoms and increase the frequency of scattering events of charge carriers, which directly leads to the resistance increase of the Pd. In this context, it is expected that the magnitude of the resistance increase is proportional to the atomic fraction of absorbed hydrogen atoms to Pd atoms, as expressed by a similar Sievert's law, namely $\Delta \mathrm{R} / \mathrm{R}_{0} \sim\left(\mathrm{pH}_{2}\right)^{1 / 2}$ [7]. Fig. 6 includes a satisfactory fit of sensitivity data to a Sievert's law over the entire $\mathrm{H}$ content range. This constitutes further evidence of Pd-H sensing in the dilute $\alpha$-hydride phase in our nanoribbon arrays for $1 \% \mathrm{H}_{2} / \mathrm{N}_{2}$ gas.

Furthermore, the nanoribbons presented here are not expected to experience buckling effects even for larger thicknesses and higher hydrogen loadings (leading to the stabilizations of the Beta-phase) due to the porous nature of the ribbons, which could thus accommodate the concurrent particle growth without introducing tensions.

\section{Conclusions}

A novel sequential combination of two nanostructuring techniques has proved successful to form palladium nanoparticle ribbons useful as transparent conductors and hydrogen sensors. The nanostructuring approach, which comprises the formation of laser-induced periodic surface structures followed by sputter gas phase aggregation oblique NP deposition, does not require any clean-room facilities, is compatible with integrated circuit processes and can be applied to soft substrates. The approach has resulted in nanoribbon arrays of palladium nanoparticles that have been demonstrated to constitute a new multifunctional material system with properties of (1) optical transparency simultaneous with electrical conduction, (2) electrical resistance anisotropy and (3) hydrogen detection capability. It is likely that the flexible nature of the PET substrate could bestow additional functionality to the material system [30].

The ripple pattern dimensions achieved by the laser treatment of the polymer PET surface are significantly large, the crest spacing being about $204 \mathrm{~nm}$ and its amplitude around $46 \mathrm{~nm}$. It has been demonstrated that such a periodic patterned surface enables self-shadowing from a beam of Pd nanoparticles in the oblique deposition process. We have shown how the ribbon width may be tailored via control of the angle of incidence of the particle beam, achieving a width down to $130 \mathrm{~nm}$. Three nanoribbon arrays of Pd NPs (diameter $5 \mathrm{~nm}$ ) on PET of differing ribbon width but constant ribbon thickness $(20 \mathrm{~nm})$ have been studied. Optical transmittance has been studied at visible wavelengths in both continuous and discontinuous Pd NP films (prepared on flat substrates) and the nanoribbon arrays. We demonstrate that transmittance in the latter is governed by the ribbon width/gap size of the array and a functional transmittance value above $80 \%$ can be achieved if the inter-ribbon distance is increased above $100 \mathrm{~nm}$. The Pd nanoribbon arrays constitute a system of anisotropic resistance whose electrical conductivity is at present insufficient for device usage. However, a comparison with other TC systems together with theoretical calculations [20] allow us to envisage how sheet resistance may be reduced by either increasing the ribbon thickness, replacing the NP by continuous ribbons (which, in turn, would require some directional material source), or both. Finally, reversible detection of hydrogen by the Pd NP arrays is observed down to an equivalent hydrogen concentration in ambient conditions of around $60 \mathrm{ppm}$, with good sensitivity, especially 
at a total pressure near to 1 bar. Based on previous studies on Pd-H nanoparticles together with our observation of a Sievert's law dependence, we conclude that our nanoribbon arrays operate in the dilute $\alpha$ hydride regime.

\section{Acknowledgements}

This work was supported by the Spanish Ministerio de Economía y Competitividad (grant numbers MAT2015-65295-R and CTQ201675880-P). E.R. thanks MINECO for a Ramón y Cajal contract (RYC2011-08069). We are grateful to Eduardo Prado, Mario Rivera, David Vela and Pablo Muñiz for technical assistance.

\section{References}

11] Y. Xia, P. Yang, Y. Sun, Y. Wu, B. Mayers, B. Gates, Y. Yin, F. Kim, H. Yan, Onedimensional nanostructures: synthesis, characterization, and applications**, Adv. Mater. 15 (2003) 353-389.

[2] G. Cao, Y. Wang, Nanostructures and Nanomaterials: Synthesis, Properties and Applications, second ed., World Scientific Publishing Co. Pte. Ltd., London, 2011.

[3] S. Pirsalami, S.M. Zebarjad, H. Daneshmanesh, An overview of metallic nanowire networks, promising building blocks for next generation transparent conductors: emergence, fundamentals and challenges, J. Electron. Mater. 46 (2017) 4707-4715.

[4] K.D. Harris, A.C. Van Popta, J.C. Sit, D.J. Broer, M.J. Brett, A birefringent and transparent electrical conductor, Adv. Funct. Mater. 18 (2008) 2147-2153.

[5] C.N. De Carvalho, G. Lavareda, E. Fortunato, P. Vilarinho, A. Amaral, ITO films deposited by rf-PERTE on unheated polymer substrates - Properties dependence on In-Sn alloy composition, Mater. Sci. Eng. B Solid-State Mater. Adv. Technol. 109 (2004) 245-248.

[6] Critical Raw Materials - European Commission, n.d. http://ec.europa.eu/growth/ sectors/raw-materials/specific-interest/critical_es (accessed 10.09.18)

[7] J.S. Noh, J.M. Lee, W. Lee, Low-dimensional palladium nanostructures for fast and reliable hydrogen gas detection, Sensors 11 (2011) 825-851.

[8] W.J. Buttner, M.B. Post, R. Burgess, C. Rivkin, An overview of hydrogen safety sensors and requirements, Int. J. Hydrogen Energy 36 (2011) 2462-2470.

[9] Y. Sakamoto, K. Takai, I. Takashima, M. Imada, Electrical resistance measurements as a function of composition of palladium - hydrogen(deuterium) systems by a gas phase method, J. Phys. Condens. Matter. 8 (1999) 3399-3411.

[10] R.K. Joshi, S. Krishnan, M. Yoshimura, A. Kumar, Pd nanoparticles and thin films for room temperature hydrogen sensor, Nanoscale Res. Lett. 4 (2009) 1191-1196.

[11] A. Pundt, Hydrogen in nano-sized metals, Adv. Eng. Mater. 6 (2004) 11-21.

[12] A. Pundt, M. Dornheim, M. Guerdane, H. Teichler, H. Ehrenberg, M.T. Reetz N.M. Jisrawi, Evidence for a cubic-to-icosahedral transition of quasi-free Pd-Hclusters controlled by the hydrogen content, Eur. Phys. J. D - At. Mol. Opt. Phys. 19 (2002) 333-337.

[13] E. Rebollar, M. Castillejo, T.A. Ezquerra, Laser induced periodic surface structures on polymer films: from fundamentals to applications, Eur. Polym. J. 73 (2015) $162-174$.

[14] I. Gnilitskyi, T.J.-Y. Derrien, Y. Levy, N.M. Bulgakova, T. Mocek, L. Orazi, Highspeed manufacturing of highly regular femtosecond laser-induced periodic surface structures: physical origin of regularity, Sci. Rep. 7 (2017).

[15] S. He, J.J. Nivas, K.K. Anoop, A. Vecchione, M. Hu, R. Bruzzese, S. Amoruso, Surface structures induced by ultrashort laser pulses: Formation mechanisms of ripples and grooves, Appl. Surf. Sci. 353 (2015) 1214-1222.

[16] T.Q. Jia, H.X. Chen, M. Huang, F.L. Zhao, J.R. Qiu, R.X. Li, Z.Z. Xu, X.K. He, J. Zhang, H. Kuroda, Formation of nanogratings on the surface of a ZnSe crystal irradiated by femtosecond laser pulses, Phys. Rev. B. 72 (2005) 125429.

[17] B. Wickman, M. Fredriksson, L. Feng, N. Lindahl, J. Hagberg, C. Langhammer, Depth probing of the hydride formation process in thin Pd films by combined electrochemistry and fiber optics-based in situ UV/vis spectroscopy, PCCP 17 (2015) 18953-18960.

[18] S. De, T.M. Higgins, P.E. Lyons, E.M. Doherty, P.N. Nirmalraj, W.J. Blau, J.J. Boland, J.N. Coleman, Silver nanowire networks as flexible, transparent, conducting films: extremely high DC to optical conductivity ratios, ACS Nano 3 (2009) 1767-1774.

[19] H. Wu, L. Hu, M.W. Rowell, D. Kong, J.J. Cha, J.R. McDonough, J. Zhu, Y. Yang, M.D. McGehee, Y. Cui, Electrospun metal nanofiber webs as high-performance transparent electrode, Nano Lett. 10 (2010) 4242-4248.

[20] P.B. Catrysse, S. Fan, Nanopatterned metallic films for use as transparent conductive electrodes in optoelectronic devices, Nano Lett. 10 (2010) 2944-2949.

[21] P.C. Hsu, D. Kong, S. Wang, H. Wang, A.J. Welch, H. Wu, Y. Cui, Electrolessly deposited electrospun metal nanowire transparent electrodes, J. Am. Chem. Soc 136 (2014) 10593-10596.

[22] S. Ye, A.R. Rathmell, Z. Chen, I.E. Stewart, B.J. Wiley, Metal nanowire networks: The next generation of transparent conductors, Adv. Mater. 26 (2014) 6670-6687.

[23] R.A. Hatton, M.R. Willis, M.A. Chesters, D. Briggs, A robust ultrathin, transparent gold electrode tailored for hole injection into organic light-emitting diodes, J. Mater. Chem. 13 (2003) 722-726.

[24] C.F. Guo, T. Sun, Q. Liu, Z. Suo, Z. Ren, Highly stretchable and transparent nanomesh electrodes made by grain boundary lithography, Nat. Commun. 5 (2014) 1-8.

[25] X.Q. Zeng, M.L. Latimer, Z.L. Xiao, S. Panuganti, U. Welp, W.K. Kwok, T. Xu, Hydrogen gas sensing with networks of ultrasmall palladium nanowires formed on filtration membranes, Nano Lett. 11 (2011) 262-268.

[26] M. Khanuja, D. Varandani, B.R. Mehta, Pulse like hydrogen sensing response in Pd nanoparticle layers, Appl. Phys. Lett. 91 (2007) 1-4.

[27] F. Yang, S.C. Kung, M. Cheng, J.C. Hemminger, R.M. Penner, Smaller is faster and more sensitive: The effect of wire size on the detection of hydrogen by single palladium nanowires, ACS Nano 4 (2010) 5233-5244.

[28] F. Rumiche, H.H. Wang, W.S. Hu, J.E. Indacochea, M.L. Wang, Anodized aluminum oxide (AAO) nanowell sensors for hydrogen detection, Sens. Actuators, B Chem. 134 (2008) 869-877.

[29] F.A. Lewis, The Palladium Hydrogen System, first ed., Academic Press Inc. Ltd, London and New York, 1967.

[30] J.J. Lee, S. Kim, J.J. Lee, D. Yang, B.C. Park, S. Ryu, I. Park, A stretchable strain sensor based on a metal nanoparticle thin film for human motion detection, Nanoscale 6 (2014) 11932-11939. 